\title{
Determination of extra virgin olive oil in quaternary mixture using FTIR spectroscopy and multivariate calibration
}

\author{
Abdul Rohman ${ }^{\mathrm{a}, \mathrm{c}, *}$ and Y.B. Che Man ${ }^{\mathrm{b}}$ \\ ${ }^{a}$ Research Center of Halal Products, Gadjah Mada University, Yogyakarta, Indonesia \\ ${ }^{\mathrm{b}}$ Halal Products Research Institute, Universiti Putra Malaysia, Serdang, Selangor, Malaysia \\ ${ }^{\mathrm{c}}$ Department of Pharmaceutical Chemistry, Faculty of Pharmacy, Gadjah Mada University, \\ Yogyakarta, Indonesia
}

\begin{abstract}
The purpose of this study was to optimize Fourier transform infrared (FTIR) spectroscopy in combination with multivariate calibrations (partial least square and principle component regression) for determination of extra virgin olive oil (EVOO) in quaternary mixture systems with grape seed oil (GSO), rice bran oil (RBO) and walnut oil (WO). FTIR spectra of EVOO in quaternary mixtures were subjected to several treatments including mean centering (MC), standard normal variate, and spectra derivatives. The combined frequency regions of $1200-900$ and $2949-2885 \mathrm{~cm}^{-1}$ were used for determination of EVOO. Using partial least square calibration, FTIR normal spectra treated with MC model give the highest values of coefficient of determination $\left(R^{2}\right)$ and the lowest values of root mean square error of calibration (RMSEC). The $R^{2}$ value obtained for the relationship between actual and FTIR predicted value of EVOO was $>0.99$ with RMSEC value of $1.55 \%$ (v/v). The developed PLS model was further used to calculate EVOO in prediction samples, and the root mean square error of prediction obtained was $3.65 \%(\mathrm{v} / \mathrm{v})$.
\end{abstract}

Keywords: FTIR spectroscopy, extra virgin olive oil, partial least square, principle component regression, quaternary mixtures

\section{Introduction}

Extra virgin olive oil (EVOO) is high value edible oils and is considered as functional food oil due to its capability to provide specific body functions [9]. Garcia-Gonzalez et al. [7] have reviewed the beneficial effects of EVOO; therefore, its analysis is highly needed in order to ensure the purity and the authenticity of EVOO. Analysis of fats and oils is typically performed by determining certain classes of fats and oil such as fatty acids, triglycerides, sterols, etc. rather than analysis of fats and oils as a whole matter. FTIR spectroscopy can be developed for analysis of fats and oils as a whole matter. Currently, FTIR spectroscopy has been considered as an attractive and powerful analytical technique for analysis of edible fats and oils [20].

The quantitative analysis of multi-component mixtures using spectroscopic-based techniques is very difficult, because the presence of the spectral overlapping among the analytes of interest in the complex mixture [8]. Currently, the use of multivariate calibrations for analysis of complex mixtures has grown

\footnotetext{
${ }^{*}$ Corresponding author: Abdul Rohman, Research Center of Halal Products, Gadjah Mada University, Yogyakarta 55281, Indonesia. Tel.: +62 2745 43120; Fax: +03 89439745; E-mail: abdulkimfar@gmail.com.
} 
fast and is receiving popularity for the quantification of edible fats and oils in the mixture due to its ability to use the complete spectral information, frequently with no excessive sample separation [2].

In the last few decades, multivariate calibrations have become a vital analytical tool in many different fields, especially in food chemistry, pharmaceutical analysis and agriculture [6]. Multivariate calibration is distinct from univariate calibration in that the experimental data in multivariate calibration consists of several variables rather than one variable in univariate calibration. The most common multivariate calibrations used are partial least square (PLS) and principal component regression (PCR) [3]. Both calibrations are based on "components" or "latent variables" which are linear combination of original variables [11].

FTIR spectroscopy combined with multivariate calibration of PLS and PCR has been developed for analysis of EVOO in binary [1,17] and ternary mixtures with other oils [18]. Besides, FTIR spectroscopy was also used for determination of animal fats in quaternary mixture [15] and for authentication of Nigella sativa seed oil [19]. However, there is no available report related to the application of FTIR spectroscopy for analysis of EVOO in quaternary mixtures with other oils. In this study, FTIR spectroscopy combined with PLS and PCR as well as coupled with several treatments was optimized for determination of EVOO in quaternary mixture systems with grape seed oil, rice bran oil and walnut oil.

\section{Material and methods}

\subsection{Sample preparation}

Extra virgin olive oil (EVOO), grape seed oil (GSO), rice bran oil (RBO) and walnut oil (WO) were purchased from the local market in Serdang, Selangor, Malaysia. The composition of the fatty acids was determined using gas chromatography with flame ionization detector as derivate of fatty acid methyl ester (FAME) as described in our previous paper [16]. The 37 FAME standards were obtained from Sigma (Aldrich, USA). The solvents and reagents used were of analytical grade.

\subsection{Calibration and validation}

The calibration samples composed of EVOO in quaternary mixture with GSO, RBO and WO in the concentration range of $0-100 \% \mathrm{v} / \mathrm{v}$ as neat form was shown in Table 1. Furthermore, a series of independent samples was also prepared as validation/prediction samples in order to evaluate the predictive ability of the developed calibration model. The composition of EVOO and others in quaternary mixtures was randomly designed in order to avoid the correlated concentration profiles [15]. All samples were analyzed using FTIR spectrometer.

\subsection{Measurement of FTIR spectra}

FTIR spectra of all evaluated samples were acquired using Nicolet 6700 from Thermo Scientific (Madison, USA) equipped with detector of deuterated triglycine sulfat (DTGS) and beam splitter composed of KBr. The OMNIC operating system (Version 7.0, Thermo Nicolet) was used for spectra measurements. Using Pasteur pipette, the samples were placed in contact with horizontal attenuated total reflectance (HATR) element (ZnSe crystal) at controlled ambient temperature $\left(25^{\circ} \mathrm{C}\right)$. FTIR spectra were collected in mid infrared region of $4000-650 \mathrm{~cm}^{-1}$ by co-adding 32 scans and at resolution of $4 \mathrm{~cm}^{-1}$. All spectra were rationed against a background of air spectrum. After each scan, a new reference air 
Table 1

The composition of EVOO, GSO, RBO and WO used in the calibration model

\begin{tabular}{|c|c|c|c|c|}
\hline Sample & EVOO & GSO & RBO & WO \\
\hline 1 & 100 & 0 & 0 & 0 \\
\hline 2 & 0 & 100 & 0 & 0 \\
\hline 3 & 0 & 0 & 100 & 0 \\
\hline 4 & 0 & 0 & 0 & 100 \\
\hline 5 & 2.5 & 2.5 & 47.5 & 47.5 \\
\hline 6 & 47.5 & 47.5 & 2.5 & 2.5 \\
\hline 7 & 45.0 & 45.0 & 5.0 & 5.0 \\
\hline 8 & 5.0 & 5.0 & 45.0 & 45.0 \\
\hline 9 & 7.5 & 10.0 & 40.0 & 42.5 \\
\hline 10 & 10.0 & 70.0 & 7.5 & 12.5 \\
\hline 11 & 70.0 & 12.5 & 10.0 & 7.5 \\
\hline 12 & 97.43 & 1.28 & 1.28 & 0 \\
\hline 13 & 1.94 & 1.94 & 3.88 & 92.22 \\
\hline 14 & 30.0 & 25.0 & 25.0 & 20.0 \\
\hline 15 & 25.0 & 20.0 & 30.0 & 25.0 \\
\hline 16 & 13.6 & 9.1 & 68.2 & 9.1 \\
\hline 17 & 10.9 & 3.2 & 82.7 & 3.2 \\
\hline 18 & 16.3 & 77.5 & 3.1 & 3.1 \\
\hline 19 & 35.44 & 30.38 & 3.8 & 30.38 \\
\hline 20 & 41.1 & 41.1 & 13.7 & 4.1 \\
\hline 21 & 3.65 & 3.65 & 19.75 & 73.17 \\
\hline 22 & 77.3 & 16.9 & 2.9 & 2.9 \\
\hline 23 & 40.0 & 40.0 & 11.25 & 8.75 \\
\hline 24 & 16.67 & 11.91 & 35.71 & 35.71 \\
\hline 25 & 4.54 & 70.45 & 20.45 & 4.54 \\
\hline 26 & 50 & 0 & 50 & 0 \\
\hline 27 & 0 & 50 & 0 & 50 \\
\hline
\end{tabular}

background spectrum was taken. These spectra were recorded as absorbance values at each data point in triplicate.

\subsection{Statistical analysis}

Multivariate calibrations (PLS and PCR) were performed using the software TQ Analyst ${ }^{\mathrm{TM}}$ (Thermo Electron Corporation). The values of coefficient of determination $\left(R^{2}\right)$ and root mean square error of calibration (RMSEC) were used as performance criteria for calibration model. The predictive ability of model was assessed using $R^{2}$ values and root mean square error of prediction (RMSEP). PLS and PCR models were further validated using "the leave-one-out cross" technique, and the root mean square error of cross validation (RMSECV) values were computed.

\section{Results and discussion}

Figure 1 exhibited FTIR spectra of four vegetable oils namely EVOO, GSO, RBO and WO measured in mid infrared region $\left(4000-650 \mathrm{~cm}^{-1}\right)$. Each bands and shoulders in FTIR spectra originated from the 


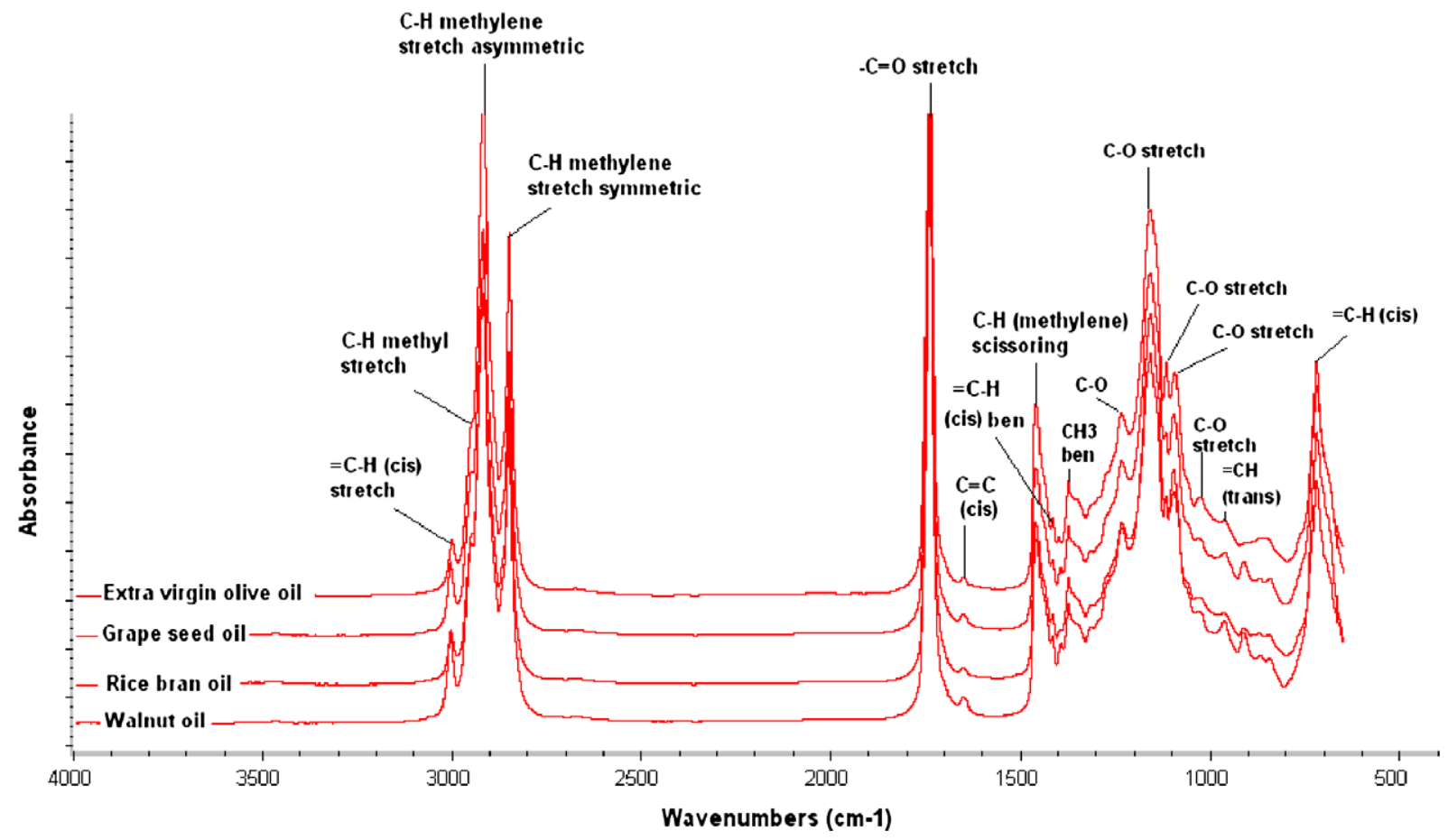

Fig. 1. FTIR spectra of EVOO, GSO, RBO and WO in mid infrared regions $\left(4000-650 \mathrm{~cm}^{-1}\right)$. (Colors are visible in the online version of the article; http://dx.doi.org/10.3233/SPE-2011-0538.)

Table 2

The composition of fatty acids in EVOO, GSO, RBO and WO

\begin{tabular}{llllrlllll}
\hline Oils $^{\ddagger}$ & \multicolumn{8}{c}{ Fatty acids $(\% \mathrm{w} / \mathrm{w})^{\dagger}$} \\
\cline { 2 - 10 } & \multicolumn{1}{l}{ C10:0 } & \multicolumn{1}{l}{ C12:0 } & \multicolumn{1}{l}{ C14:0 } & \multicolumn{1}{c}{ C16:0 } & \multicolumn{1}{l}{ C18:0 } & C18:1 & C18:2 & C18:3 & C20:0 \\
\hline EVOO & $0.01 \pm 0.00$ & $0.01 \pm 0.00$ & $0.01 \pm 0.00$ & $10.78 \pm 0.25$ & $3.30 \pm 0.13$ & $74.98 \pm 1.72$ & $7.77 \pm 0.35$ & $0.63 \pm 0.04$ & $0.43 \pm 0.03$ \\
GSO & $0.05 \pm 0.00$ & $0.01 \pm 0.00$ & $0.01 \pm 0.00$ & $7.87 \pm 0.12$ & $3.27 \pm 0.09$ & $21.16 \pm 1.07$ & $64.02 \pm 1.24$ & $0.77 \pm 0.02$ & $0.23 \pm 0.01$ \\
RBO & $0.01 \pm 0.00$ & $0.01 \pm 0.00$ & $0.35 \pm 0.01$ & $18.85 \pm 1.02$ & $0.50 \pm 0.04$ & $43.17 \pm 1.28$ & $31.74 \pm 0.84$ & $0.85 \pm 0.05$ & $1.34 \pm 0.12$ \\
WO & $0.03 \pm 0.00$ & $0.01 \pm 0.00$ & $0.01 \pm 0.00$ & $7.11 \pm 0.12$ & $3.24 \pm 0.11$ & $19.75 \pm 0.32$ & $60.34 \pm 0.74$ & $1.28 \pm 0.21$ & $0.49 \pm 0.01$ \\
\hline
\end{tabular}

$\dagger$ Each value in the table represents the means of triplicate analysis; SD is given in after \pm ; ${ }^{\ddagger}$ EVOO - extra virgin olive oil; GSO - grape seed oil; RBO - rice brain oil; WO - walnut oil.

functional groups responsible for infrared absorption. The interpretation of each peak and shoulder in FTIR spectra was also included in Fig. 1. Peaks in the spectra are very similar since the studied vegetable oils were mainly composed from triglyceride with certain fatty acids (Table 2). However, upon scrutiny, the spectra of studied oils reveal a bit differences in terms of band intensities and the exact frequencies at which the maximum absorbance are generated in each oils.

Analysis of EVOO in quaternary systems with GSO, RBO and WO was performed using multivariate calibrations of PLS and PCR algorithms. In multivariate calibrations, the samples of vegetable oils were independently separated into calibration and validation data sets, respectively. The separation into data sets is carried out in order to get the similar standard deviation and average values so that both data sets spanned the working range of the studied vegetable oils [14]. The overlay of calibration spectra 


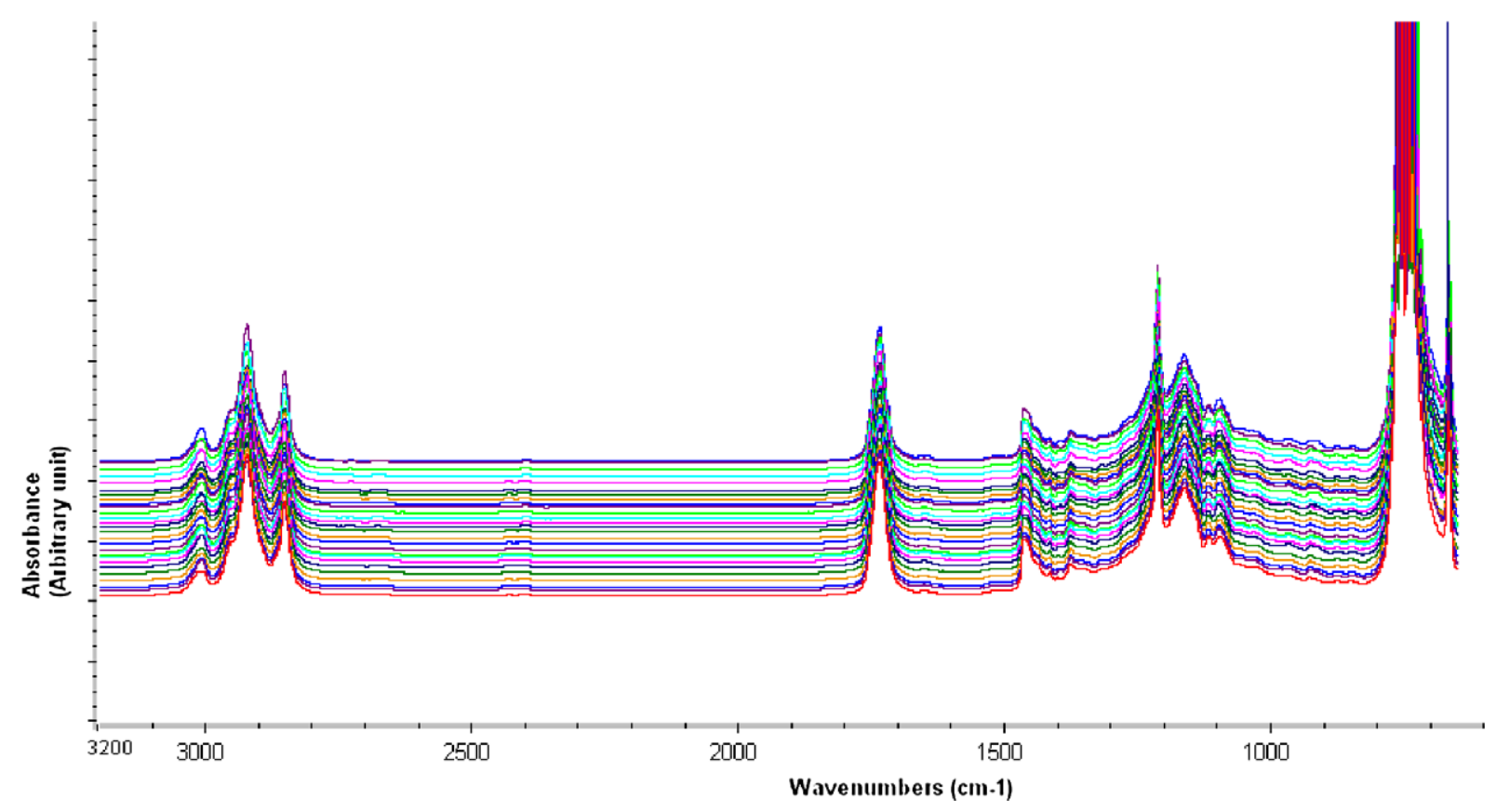

Fig. 2. The overlay of FTIR calibration spectra of the mixtures of EVOO, GSO, RBO and WO. (Colors are visible in the online version of the article; http://dx.doi.org/10.3233/SPE-2011-0538.)

composed from EVOO, GSO, RBO and WO in quaternary mixtures with the composition as shown in Table 1 was depicted in Fig. 2.

The first optimization for determination of EVOO in quaternary systems with GSO, RBO and WO was carried by selecting the frequency regions in such a way that the analyst describes the most characteristics oil components to be determined. PLS and PCR can be applied for the analysis of component of interest at wide FTIR spectral regions rather than specific regions [5]. For these reasons, some frequency regions were evaluated for its performance. The spectral regions exploited for such analysis are optimized in such a way that it gives the highest value of $R^{2}$ and the lowest values of RMSEC. The spectral regions between 4000-3050 and $2750-1850 \mathrm{~cm}^{-1}$ were removed because they do not contain useful information regarding to the quantification of EVOO. In addition, they contribute to the instrumental noise.

Table 3 compiled the PLS and PCR performances for quantification of EVOO and others (GSO, RBO and WO) at some frequency regions. Relying on the highest values of $R^{2}$ and the lowest values of RMSEC in Table 3, it can be stated that EVOO in quaternary systems with GSO, RBO and WO was better analyzed at combined spectral regions of $1200-900$ and $2949-2885 \mathrm{~cm}^{-1}$. In addition, PLS model was more favorable to be used in the quantification of oils compared with PCR. The number of factors or principal components (PCs) used in the model was chosen to optimize the performance of PLS model and to minimize errors in the model caused by under-fitting and over-fitting the data [12].

The next optimization was performed using several treatments, namely mean centering (MC) which eliminates the common information in FTIR spectra; standard normal variate (SNV) which scales FTIR spectral data in order to compensate the pathlength differences; and derivatization using Savitzky-Golay first and second derivatives [21]. The first derivative removes the common intensity effect of FTIR spectral and can simplify the baseline selection. Meanwhile, the second derivative can eliminate the slope effect. However, derivation treatments can strongly affect the measurement sensitivity [4]. 
Table 3

PLS and PCR performances at some frequency regions for determination of EVOO, GSO, RBO and WO in quaternary mixtures

\begin{tabular}{|c|c|c|c|c|}
\hline Frequency regions $\left(\mathrm{cm}^{-1}\right)$ & Analytes & Multivariate calibration & $R^{2}$ & $\operatorname{RMSEC}(\% \mathrm{v} / \mathrm{v})$ \\
\hline \multirow[t]{8}{*}{$3050-2750$ and $1850-650$} & EVOO & PLS & 0.520 & 25.1 \\
\hline & & PCR & 0.508 & 25.3 \\
\hline & GSO & PLS & 0.591 & 22.4 \\
\hline & & PCR & 0.634 & 21.5 \\
\hline & $\mathrm{RBO}$ & PLS & 0.574 & 21.9 \\
\hline & & PCR & 0.625 & 20.9 \\
\hline & WO & PLS & 0.504 & 24.5 \\
\hline & & PCR & 0.492 & 24.7 \\
\hline \multirow[t]{8}{*}{ 2949-2885 } & EVOO & PLS & 0.821 & 16.8 \\
\hline & & PCR & 0.928 & 10.9 \\
\hline & GSO & PLS & 0.336 & 26.2 \\
\hline & & PCR & 0.769 & 17.8 \\
\hline & $\mathrm{RBO}$ & PLS & 0.279 & 25.7 \\
\hline & & PCR & 0.786 & 16.5 \\
\hline & WO & PLS & 0.731 & 19.4 \\
\hline & & PCR & 0.858 & 14.5 \\
\hline \multirow[t]{8}{*}{$1200-900$} & EVOO & PLS & 0.999 & 1.41 \\
\hline & & PCR & 0.997 & 2.13 \\
\hline & GSO & PLS & 0.995 & 2.71 \\
\hline & & PCR & 0.988 & 4.15 \\
\hline & $\mathrm{RBO}$ & PLS & 0.999 & 1.21 \\
\hline & & PCR & 0.993 & 2.94 \\
\hline & WO & PLS & 0.997 & 1.95 \\
\hline & & PCR & 0.995 & 2.82 \\
\hline \multirow{8}{*}{ 1200-900 and 2949-2885 } & $E V O O$ & $P L S$ & 0.998 & 1.55 \\
\hline & & PCR & 0.994 & 3.13 \\
\hline & GSO & $P L S$ & 0.998 & 1.57 \\
\hline & & PCR & 0.971 & 6.63 \\
\hline & $R B O$ & $P L S$ & 0.995 & 2.61 \\
\hline & & PCR & 0.988 & 4.10 \\
\hline & $W O$ & $P L S$ & 0.999 & 0.172 \\
\hline & & PCR & 0.983 & 5.20 \\
\hline
\end{tabular}

Note: The frequency regions and multivariate calibration chosen for quantification were marked with italic.

Table 4 listed the performance of multivariate calibrations for determination of EVOO and others (GSO, RBO and WO) in quaternary systems using the combination of spectral treatments at optimized frequencies of 1200-900 and 2949-2885 $\mathrm{cm}^{-1}$ and PLS model. FTIR normal spectra treated with MC offers the high value of $R^{2}$ and the low value of RMSEC. Consequently, this treatment was selected for quantification of EVOO and others. Figure 3 revealed the PLS scatter plot for the relationship between actual value ( $x$-axis) and FTIR predicted value ( $y$-axis) of EVOO as well as GSO, RBO and WO using FTIR normal spectra treated with MC.

The performance of the developed PLS model was cross-validated using "leave-one out" technique. In cross validation, one of the calibration samples was removed from the model and the residual samples were used to build new PLS model. Subsequently, the taken out sample is computed using the new PLS regression. This manner was recurred, leaving each sample out in turn [13]. Using the optimized 
Table 4

The performance of multivariate calibration for quantification of EVOO in quaternary mixture with GSO, RBO and WO using PLS model ${ }^{\dagger}$

\begin{tabular}{|c|c|c|c|c|c|}
\hline Pretreatments & Analytes & Factors & $R^{2}$ & RMSEC (\% v/v) & $\operatorname{RMSEP}(\% \mathrm{v} / \mathrm{v})$ \\
\hline \multirow[t]{4}{*}{ Normal $+M C$} & $E V O O$ & 6 & 0.998 & 1.55 & 3.65 \\
\hline & GSO & 9 & 0.999 & 0.505 & 3.52 \\
\hline & $R B O$ & 6 & 0.997 & 1.95 & 4.31 \\
\hline & WO & 9 & 0.999 & 0.346 & 3.39 \\
\hline \multirow[t]{4}{*}{$\mathrm{FD}+\mathrm{MC}$} & EVOO & 5 & 0.998 & 1.74 & 9.76 \\
\hline & GSO & 9 & 0.999 & 0.243 & 11.0 \\
\hline & $\mathrm{RBO}$ & 7 & 0.999 & 0.967 & 13.8 \\
\hline & WO & 9 & 0.999 & 0.169 & 8.43 \\
\hline \multirow[t]{4}{*}{$\mathrm{SD}+\mathrm{MC}$} & EVOO & 4 & 0.994 & 3.27 & 24.4 \\
\hline & GSO & 1 & 0.357 & 25.9 & 27.4 \\
\hline & $\mathrm{RBO}$ & 9 & 0.999 & 0.13 & 36.1 \\
\hline & WO & 1 & 0.767 & 18.2 & 16.7 \\
\hline \multirow[t]{4}{*}{ Normal + SNV } & EVOO & 7 & 0.997 & 2.04 & 7.89 \\
\hline & GSO & 7 & 0.993 & 3.26 & 7.31 \\
\hline & $\mathrm{RBO}$ & 6 & 0.992 & 3.20 & 11.6 \\
\hline & WO & 7 & 0.995 & 2.75 & 6.61 \\
\hline \multirow[t]{4}{*}{$\mathrm{MD}+\mathrm{SNV}$} & EVOO & 7 & 0.999 & 0.581 & 9.47 \\
\hline & GSO & 9 & 0.999 & 0.347 & 13.0 \\
\hline & $\mathrm{RBO}$ & 4 & 0.989 & 3.93 & 15.5 \\
\hline & WO & 5 & 0.995 & 2.82 & 11.1 \\
\hline \multirow[t]{4}{*}{$\mathrm{SD}+\mathrm{SNV}$} & EVOO & 3 & 0.960 & 8.20 & 23.7 \\
\hline & GSO & 2 & 0.455 & 24.7 & 27.0 \\
\hline & $\mathrm{RBO}$ & 3 & 0.883 & 12.6 & 34.9 \\
\hline & WO & 2 & 0.757 & 18.5 & 18.8 \\
\hline
\end{tabular}

${ }^{\dagger} \mathrm{MC}$ - mean centering; FD - first derivative; SD - second derivative; SNV - standard normal variate. The spectral treatment hosen was marked with italic.

condition, the RMSECV values obtained for EVOO, GSO, RBO and WO were 3.78, 7.62, 4.78 and $5.45 \%(\mathrm{v} / \mathrm{v})$, respectively. The low RMSECV value indicated the good performance of the model.

The PLS calibration model was further used to predict the prediction/validation samples composed of EVOO, GSO, RBO and WO in quaternary system. The predictive ability of PLS was assessed by $R^{2}$ and RMSEP values. The $R^{2}$ values obtained for the relationship between actual value ( $x$-axis) and FTIR predicted values ( $y$-axis) in prediction samples were $>0.99$. In addition, the RMSEP values obtained were 3.65, 3.52, 4.31 and 3.39\% (v/v) for EVOO, GSO, RBO and WO, respectively. From these results, it can be deduced that FTIR spectroscopy combined with appropriate multivariate calibration and spectral treatment is well suited for analysis of edible fats and oils in multi-components, which offers some advantages, i.e., rapid, ease in instrumental handling, and no excessive sample preparation. In addition, the use of hazardous reagents and solvents can be avoided; therefore, the application of FTIR spectroscopy for such analysis can be considered as "green analytical technique".

\section{Conclusions}

FTIR spectroscopy combined with PLS model with normal spectra and mean centering treatment can be exploited for determination of EVOO, GSO, RBO and WO in complex mixtures. The developed 

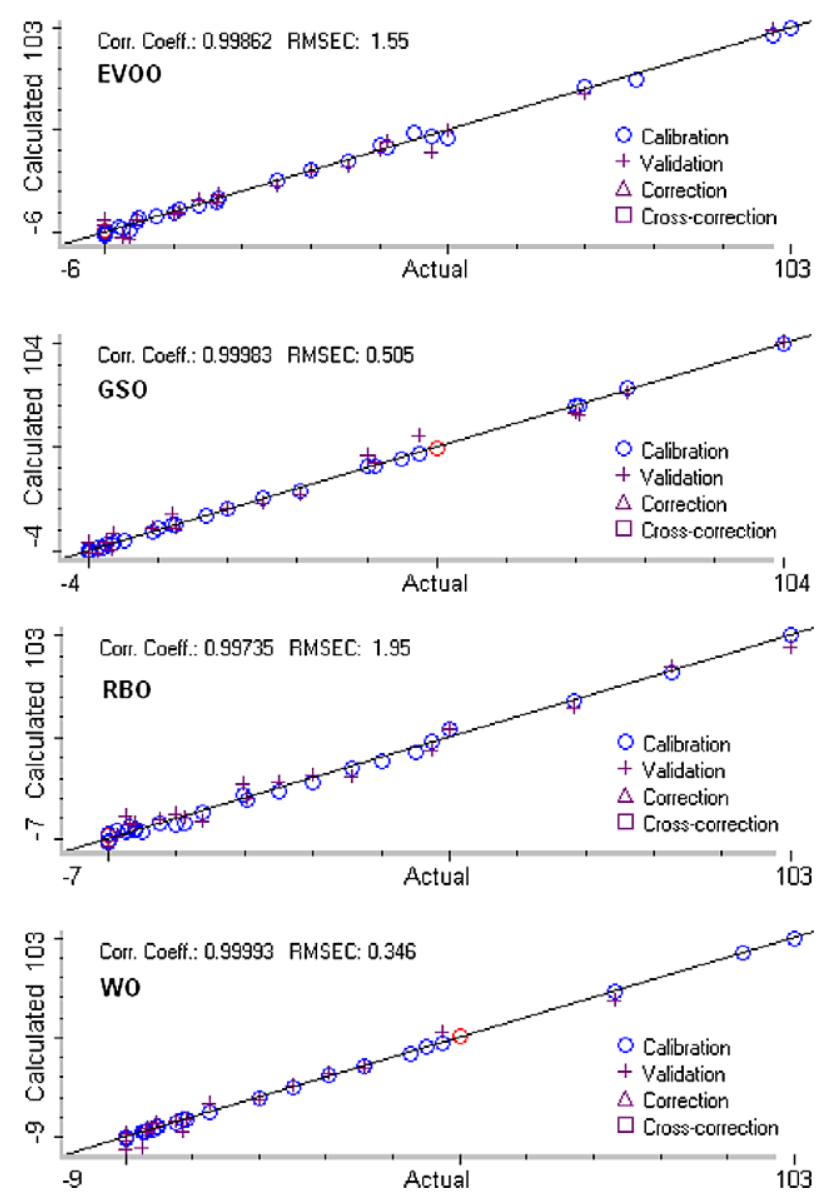

Fig. 3. The scatter plot for the relationship between actual value ( $x$-axis) and FTIR predicted value ( $y$-axis) of EVOO, GSO, RBO and WO using PLS model with normal spectra and mean centering treatment. (Colors are visible in the online version of the article; http://dx.doi.org/10.3233/SPE-2011-0538.)

method can be extended to be used in authentication study, in which the presence of low value oils such as GSO, RBO and WO in EVOO should be appropriately determined using this technique.

\section{Acknowledgements}

The first author acknowledges to The Ministry of The National Education, Republic of Indonesia for its scholarship to pursue PhD program in Halal Products Research Institute, Universiti Putra Malaysia (UPM), Malaysia.

\section{References}

[1] M.A. Alam and S.F. Hamid, J. Appl. Sci. Res. 73 (2007), 102-108.

[2] S.R.C. Andrade, I.S. Scarminio, M.M.F. Nery and A.C. de Oliveira, J. Pharm Biomed. Anal. 33 (2003), 655-665.

[3] R.G. Brereton, Applied Chemometrics for Scientist, 1st edn, Wiley, Chichester, UK, 2007. 
[4] F. Cadet and M. de la Guardia, Infrared quantitative analysis, in: Encyclopedia of Analytical Chemistry, R.A. Meyers, ed., Wiley, New York, 2001, pp. 1-26.

[5] N.M. Faber and R. Rajko, Anal. Chim. Acta 595 (2007), 98-106.

[6] M. Forina, S. Lanteri and M. Casale, J. Chromatogr. A. 118 (2007), 61-93.

[7] D.L. García-González, R. Aparicio-Ruiz and R. Aparicio, Eur. J. Lipid Sci. Technol. 110 (2008), 602-607.

[8] B. Gu, J. Wang, X. Zhou, X. Wu, F. Liu and Y. Li, J. Enviroment. Sci. Health A 33 (1998), 1419-1436.

[9] S. Kaur and M. Das, Food Sci. Biotechnol. 20 (2011), 861-875.

[10] M.R. Khoshayand, H. Abdollahi, M. Shariatpanahi, A. Saadatfard and A. Mohammadi, Spectrochim. Acta Part A 70 (2008), 491-499.

[11] R. Leardi, Grasas y Aceites 53 (2002), 115-127.

[12] M. Martens and H. Martens, Statistical Procedures in Food Research, Elsevier, London, 1986.

[13] J.N. Millerand J.C. Miller, Statistics and Chemometrics for Analytical Chemistry, 5th edn, Pearson Education Ltd, Edinburgh Gate Harlow, 2005.

[14] B. Muik, B. Lendl and A. Molina-Diaz, Anal. Bioanal. Chem. 379 (2004), 35-41.

[15] A.F. Nurrulhidayah, Y.B. Che Man, H.A. Alkahtani and A. Rohman, Spectroscopy - Biomed. Applications 25 (2011), $243-250$.

[16] A. Rohman and Y.B. Che Man, J. Food Lipids 16 (2009), 618-628.

[17] A. Rohman and Y.B. Che Man, Food Res. Int. 43 (2010), 886-892.

[18] A. Rohman and Y.B. Che Man, Food Anal. Methods 4 (2011), 155-162.

[19] A. Rohman and Y.B. Che Man, Spectroscopy - Biomed. Applications 25 (2011), 169-176.

[20] F.R. van de Voort, A. Ghetler, D.L. García-González and Y.D. Li, Food Anal. Methods 1 (2008), 153-163.

[21] L. Wang, F.S.C. Lee, X. Wang and Y. He, Food Chem. 95 (2006), 529-536. 


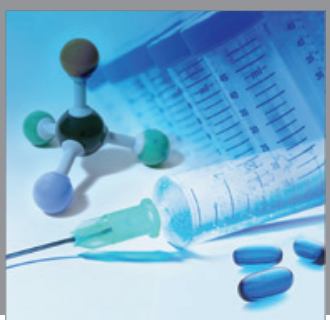

International Journal of

Medicinal Chemistry

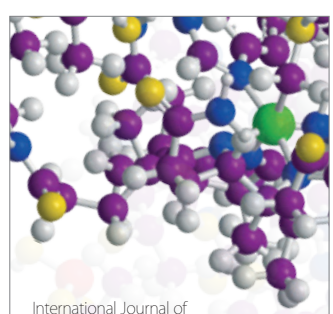

Carbohydrate Chemistry

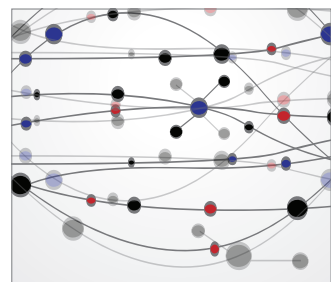

The Scientific World Journal
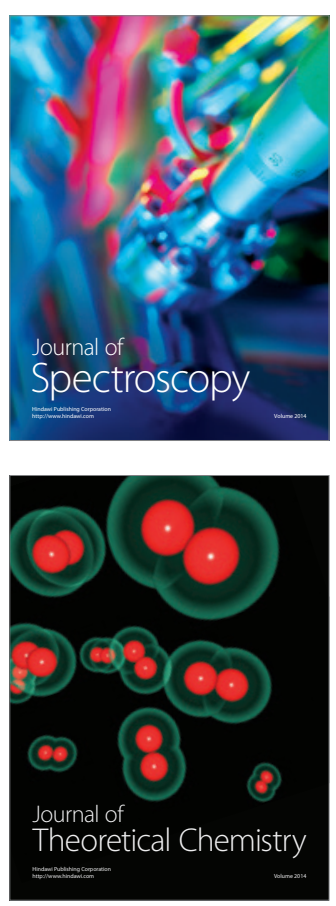
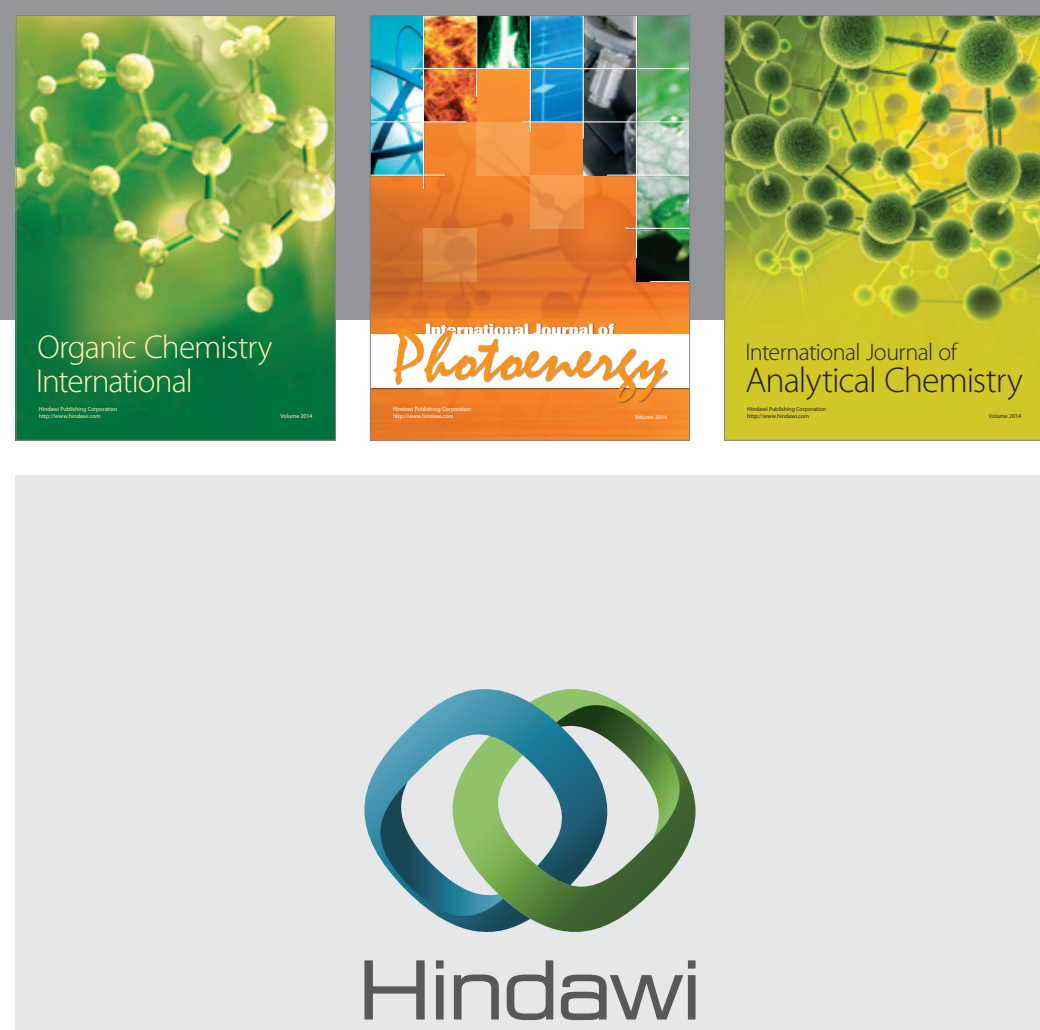

Submit your manuscripts at

http://www.hindawi.com
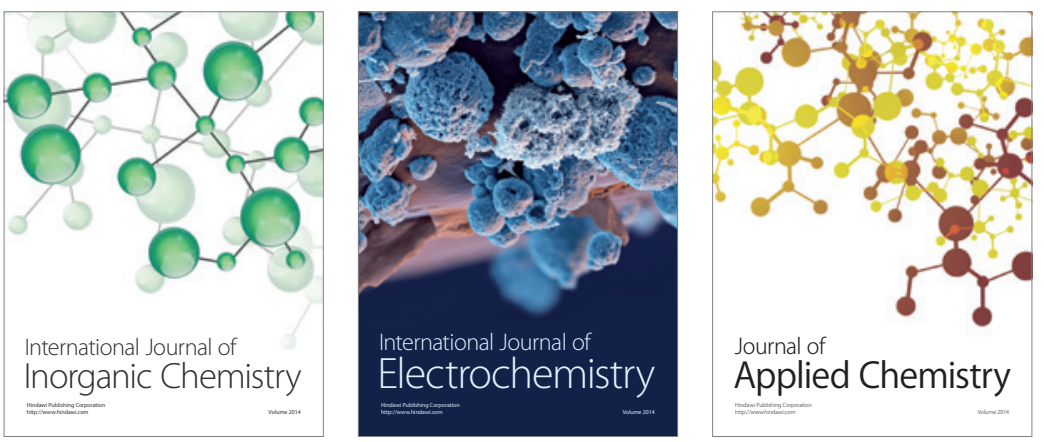

Journal of

Applied Chemistry
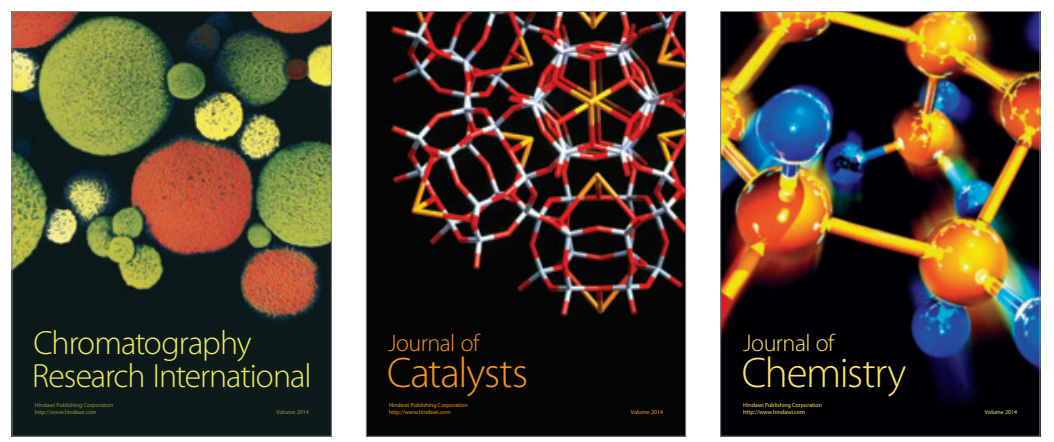
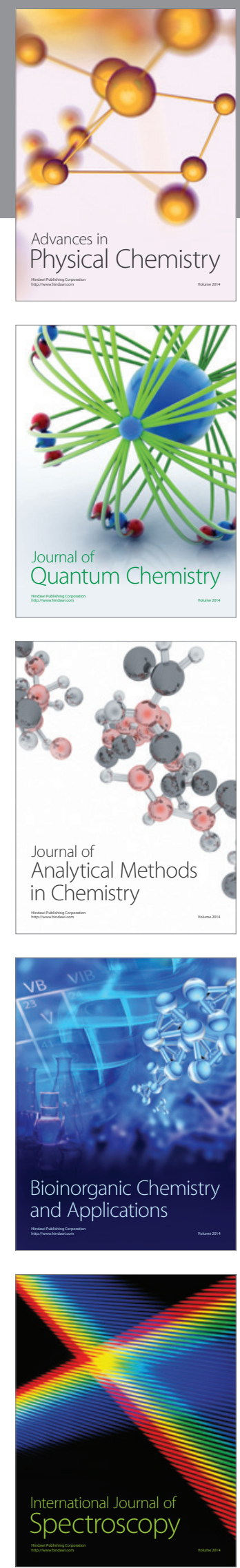